\title{
AGAPE, an experiment to detect MACHO's in the direction of the Andromeda galaxy
}

\author{
R. Ansari ${ }^{a}$, M. Aurière ${ }^{b}$, P. Baillon ${ }^{c}$, A. Bouquet ${ }^{d}$, G. Coupinot $^{b}$, \\ C. Coutures ${ }^{e}$, C. Ghesquière ${ }^{f}$, Y. Giraud-Héraud ${ }^{f}$, P. Gondolo $^{d}$, J. Hecquet ${ }^{b}$, \\ J. Kaplan ${ }^{d}$, A.L. Melchior ${ }^{d}$, M. Moniez ${ }^{a}$, J.P. Picat ${ }^{b}$ and G. Soucail ${ }^{b}$ \\ ${ }^{a}$ LAL, Université Paris Sud, Orsay, France, \\ ${ }^{b}$ Observatoire Midi-Pyrénées Bagnères de Bigorre et Toulouse, France, \\ ${ }^{c}$ CERN, Genève, Switzerland, ${ }^{d}$ LPTHE, Universités Paris 6 et 7, France, \\ ${ }^{e}$ DAPNIA, CEN Saclay, France, ${ }^{f}$ LPC Collège de France, Paris, France.
}

Presented at the 17th Texas Symposium on Relativistic Astrophysics, München germany, December 94, by J. Kaplan.

PAR-LPTHE 95-07

The M31 galaxy in Andromeda is the nearest large galaxy after the Small and Large Magellanic Clouds. It is a giant galaxy, roughly 2 times as large as our Milky Way, and has its own halo. As pointed by A. Crotts [1] and independantly by some of us [3] M31 provides a rich field of stars to search for MACHO's in galactic halos by gravitational microlensing [⿰]月 M31 is a target complementary to the Large Magellanic Cloud and the galactic bulge wich are used by the three current experiments 4, 目, 6. It is complementary in that it allows to probe the halo of our galaxy in a direction very different from that of the LMC. Moreover, the fact that M31 has its own halo and is tilted with respect to the line of sight provides a very interesting signature : assuming an approximately spherical halo for M31, the far side of the disk lies behind a larger amount of M31 dark matter, therefore more microlensing events are expected on the far side of the disk. Such an asymmetry could not be faked by variable stars 1 .

In other words, M31 seems very appropriate to detect brown dwarfs through microlensing. However, as very few stars of M31 are resolved, we had to develop an approach to look for microlensing by monitoring the pixels of a CCD, rather than individual stars 22. The AGAPE collaboration has set out to implement this idea.

\section{MONITORING PIXELS}

In the case of a crowded field such as M31, the light flux $F_{\text {pixel }}$ on a pixel comes from the many stars in and around it, plus the sky background. The light flux of an individual star, $F_{\text {star }}$, is spread among all pixels of the seeing spot and only a fraction of this light, $F_{\text {pixel }}=\{$ seeing fraction $\} \times F_{\text {star }}$, reaches the central pixel. If the star luminosity is amplified by a factor $A$, the pixel flux increases by :

$$
\Delta F_{\text {pixel }}=(A-1)\{\text { seeing fraction }\} F_{\text {star }}
$$

The amplification of the star luminosity allows an event to be detected if the flux on the brightest pixel rises sufficiently high above its rms fluctuation $\sigma_{\text {pixel }}$ :

$$
\Delta F_{\text {pixel }}>Q \sigma_{\text {pixel }}
$$

Typically, in our simulations, we require $Q$ to be larger than 3 during 3 consecutive exposures and larger than 5 for at least one of them. 


\section{EXPECTED STATISTICS.}

We have performed numerical simulations using the above detection criterium. As anticipated, the number of events we expect to be able to detect depends strongly both on the stability of the pixel and on the average seeing. The numbers in Table 1 below assume a two meter telescope, a field of view of 60 by 20 arcminutes (planed for a second generation experiment) centered on the center of M31, 1 arcsecond pixels, 30 minutes exposures, and 120 consecutive nights. The brown dwarf mass is taken to be $0.08 M_{\odot}$, and we assume a standard halo (see reference[2] for instance) with a local dark matter density of $0.3 \mathrm{GeV} / \mathrm{cm}^{3}$ $\left(0.0075 M_{\odot} / p c^{3}\right)$ and a core radius of $5 \mathrm{kpc}$.

Table 1: Expected number of events under various observing conditions

\begin{tabular}{lrrrr}
\hline seeing & $\sigma_{\text {pixel }} / F_{\text {pixel }}$ & $\begin{array}{c}\text { number of } \\
\text { galactic events }\end{array}$ & $\begin{array}{r}\text { number of } \\
\text { M31 events }\end{array}$ & total \\
\hline $1 "$ & $2 \%$ & 9 & 15 & 24 \\
& $1 \%$ & 15 & 29 & 44 \\
\hline $2 "$ & 27 & 50 & 77 \\
\hline $0.5 \%$ & $3 \%$ & 10 & 7 \\
& $2 \%$ & 7 & 21 & 32 \\
\hline
\end{tabular}

These numbers have to be compared with the 2 events per year expected by the EROS collaboration for brown dwarfs with masses of order $0.1 M_{\odot}$.

It is clear from Table 1 that the number of detectable events depends crucially on the relative flux fluctuation on the pixel, $\sigma_{\text {pixel }} / F_{\text {pixel }}$. To study the feasibility of the experiment, we have analyzed these pixel fluctuations in three series of real data (Table 2) :

- 1) 82 images of the Large Magellanic Cloud (LMC) taken by the EROS collaboration

- 2) 26 images of M31 taken with the one meter telescope at Pic du Midi, in collaboration with F. Colas (Bureau des longitudes, Paris) and J. Lecacheux (DESPA, Meudon)

Table 2: The mean relative fluctuation obtained for the three series of images listed above. The numbers in the first column refer to the numbers in the list.

\begin{tabular}{llcc}
\hline Images & $\begin{array}{l}\text { mirror size } \\
(\text { meter })\end{array}$ & $\begin{array}{c}\text { pixel size } \\
(\operatorname{arcsec})\end{array}$ & relative fluctuation \\
\hline LMC EROS $(\bullet 1)$ & 0.4 & 1.15 & $3 \%$ \\
M31 Pic $(\bullet 2)$ & 1 & 0.7 & $1 \%$ \\
M31 Pic $(\bullet 3)$ & 2 & 0.25 & $0.7 \%$ \\
M31 Pic $(\bullet 3)$ & 2 & 1 ("superpixels") & $0.23 \%$ \\
\hline
\end{tabular}

- 3) 4 images of M31 taken by E. Davoust (OMP, Toulouse) with the $2 \mathrm{~m}$ telescope at Pic du Midi. In this case, the angular size of the pixels is very small (0.23"), and we have also considered a rearrangement in $4 \times 4$ "superpixels".

The results given in Table 2 clearly show that the required photometric stability of pixels can be reached. Moreover, the analysis of the third series of data shows that pixels small compared to the seeing allow an efficient matching between images, whereas, once images are matched, superpixels are more appropriate for a stable photometry.

DISCRIMINATING AGAINST VARIABLE STARS AND OTHER VARIABILITIES

Variable stars should be the main background. The usual tools to discriminate against this background are available (symmetry, unicity, achromaticity of the light curve). Still, some points particular to our approach are discussed below. 
Achromaticity. At first sight, one would think that there is no achromaticity as a star rising above a background of a different color will cause a color variation of the pixels involved. However, it is easy to show that when a star rises above the background in two color bands (say red and blue) then the ratio

$$
\frac{\left(F_{\text {pixel }}-\left\langle F_{\text {pixel }}\right\rangle\right)_{\text {red }}}{\left(F_{\text {pixel }}-\left\langle F_{\text {pixel }}\right\rangle\right)_{\text {blue }}}=\frac{\left.F_{\text {star }}\right|_{\text {red }}}{\left.F_{\text {star }}\right|_{\text {blue }}}
$$

is constant in time during a microlensing event.

High amplifications. To rise above the background an unresolved star needs a rather high amplification $(\langle A\rangle \sim 6)$, which will exclude most variable stars.

On the other hand small secondary maxima indicating unstable stars with occasional strong flares will not be discriminated. Further study is required in this respect.

\section{THE FIRST RUN OF AGAPE}

We were given 57 half nights of observation on the 2 meter telescope "Bernard Lyot" at Observatoire du Pic du Midi in the French Pyrénées, from September 29 to November 24 1994. The field was $8^{\prime} \times 8^{\prime}$ only, covered by 4 exposures on a $800 \times 800$ thin Tektronix CCD camera with pixels 0.3 " wide.

The data of this prototype run are currently under treatment. A key step of this treatment is the alignement of successive images both in position and in photometry. The photometric alignement is performed by linearly transforming the light flux of one image in such a way that the mean flux and the variance of the transformed image matches those of some reference image. The result of this alignement between images is illustrated in figure 1. After alignement the dispersion of the relative difference between the two images is $1.6 \%$. This preliminary result is very encouraging as our alignement procedures are not yet optimized.

AKNOWLEDGMENTS We thank The EROS collaboration, and E. Davoust who allowed us to use their data, as well as F. Colas, and J. Lecacheux with whom we took data on the 1 meter telescope at Pic du Midi. The help of F. Colas during our first observation run has been particularly appreciated.

\section{References}

[1] Crotts A. P. S. 1992. ApJ. 399; L43.

[2] Baillon P. Bouquet A. Giraud-Héraud Y. \& Kaplan J. 1993. A\&A 277; 1.

[3] Paczyński B. 1986. ApJ. 304; 1.

[4] Alcock et al., 1993. Nature 365; 621, and these proceedings.

[5] Aubourg E. et al. 1993. Nature 365; 623.

[6] Udalski A. et al. 1993. Acta Astronomica 43; 289. 

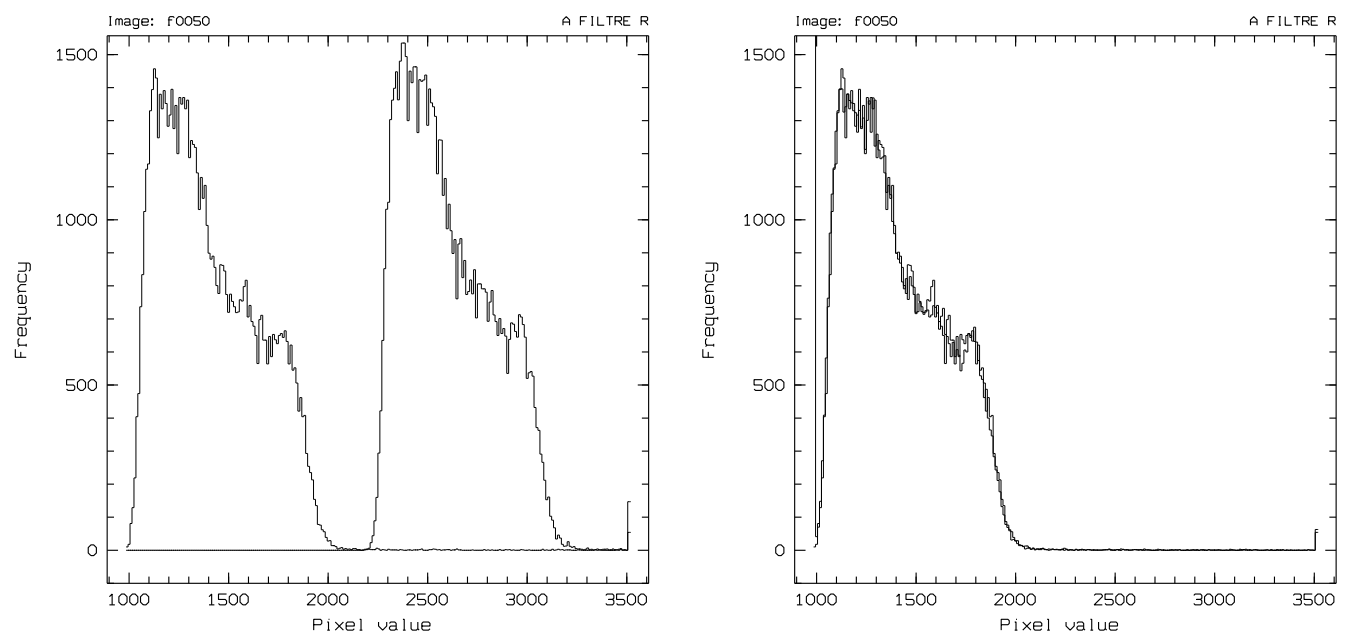

a

$\mathrm{b}$

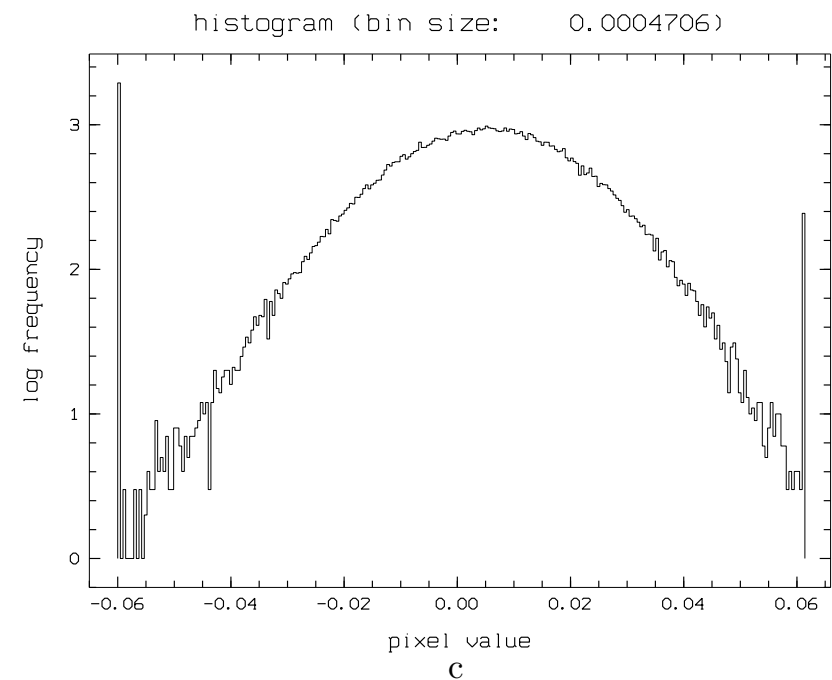

Figure 1: photometric alignement of two different images of the same field. Histogramms of pixel flux of two images, a: before treatment, b: after photometric aligement. c: Histogram of the difference of pixel fluxes between the two images, after photometric alignement. 